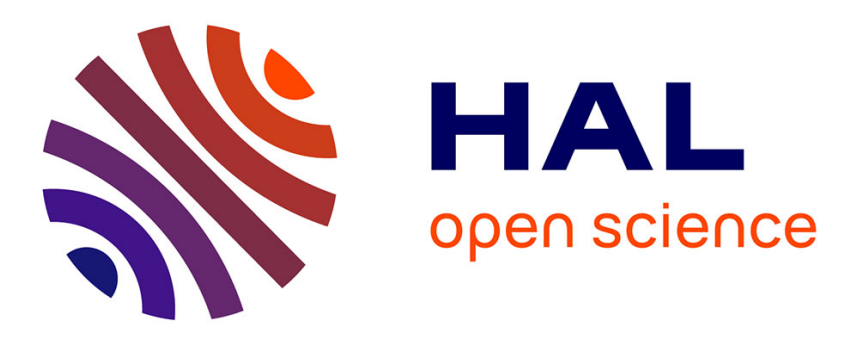

\title{
Temporal diffeomorphic Free Form Deformation to quantify changes induced by left and right bundle branch block and pacing
}

\author{
G Piella, A Porras, M de Craene, Nicolas Duchateau, A Frangi
}

\section{To cite this version:}

G Piella, A Porras, M de Craene, Nicolas Duchateau, A Frangi. Temporal diffeomorphic Free Form Deformation to quantify changes induced by left and right bundle branch block and pacing. Statistical Atlases and Computational Models of the Heart (STACOM), MICCAI'12 Workshop, 2012, Nice, France. pp.134-141, 10.1007/978-3-642-36961-2_16. hal-02320720

\section{HAL Id: hal-02320720 \\ https://hal.science/hal-02320720}

Submitted on 19 Oct 2019

HAL is a multi-disciplinary open access archive for the deposit and dissemination of scientific research documents, whether they are published or not. The documents may come from teaching and research institutions in France or abroad, or from public or private research centers.
L'archive ouverte pluridisciplinaire HAL, est destinée au dépôt et à la diffusion de documents scientifiques de niveau recherche, publiés ou non, émanant des établissements d'enseignement et de recherche français ou étrangers, des laboratoires publics ou privés. 


\title{
Temporal diffeomorphic Free Form Deformation to quantify changes induced by left and right bundle branch block and pacing
}

\author{
G. Piella ${ }^{a, b}$, A. R. Porras ${ }^{a, b}$, M. De Craene ${ }^{c}$, N. Duchateau ${ }^{d}$, \\ and A. F. Frangi ${ }^{a, b, e}$ \\ CISTIB, ${ }^{a}$ DTIC, Universitat Pompeu Fabra, and ${ }^{b}$ CIBER-BBN, Barcelona, Spain. \\ ${ }^{c}$ Philips Research, Medisys, Suresnes, France. \\ ${ }^{d}$ Hospital Clínic, IDIBAPS, Universitat de Barcelona, Spain. \\ ${ }^{e}$ University Of Sheffield, United Kingdom.
}

\begin{abstract}
This paper presents motion and deformation quantification results obtained from synthetic and in vitro phantom data provided by the second cardiac Motion Analysis Challenge at STACOM-MICCAI. We applied the Temporal Diffeomorphic Free Form Deformation (TDFFD) algorithm to the datasets. This algorithm builds upon a diffeomorphic version of the FFD, to provide a $3 D+t$ continuous and differentiable transform. The similarity metric includes a comparison between consecutive images, and between a reference and each of the following images. Motion and strain accuracy were evaluated on synthetic 3D ultrasound sequences with known ground truth motion. Experiments were also conducted on in vitro acquisitions.
\end{abstract}

\section{Introduction}

Estimation of motion and strain gives insight into cardiac function by quantifying how a given pathology affects global and local deformation of the myocardium. This provides useful information for diagnosis, treatment, and follow-up of cardiac diseases. In clinical routine, motion and strain are often obtained from ultrasound (US) images because it is safe, non-invasive and cost-effective. Although 3D acquisition systems are increasingly used, 3D US images have lower quality and temporal resolution than the $2 \mathrm{D}$ ones, thus making their processing more challenging. Nonetheless, processing $3 \mathrm{D}$ has the advantage of providing the whole motion and deformation of heart, unlike 2D for which only the projection of these parameters on the observation plane is available. This is particularly important for an accurate quantification, due to the cardiac architecture (e.g., fiber orientation) which imposes by itself motion and deformation to be 3D.

In this paper, we use the Temporal Diffeomorphic Free Form Deformation (TDFFD) algorithm $[1,2]$ and apply it to the synthetic and in vitro data provided by the second cardiac Motion Analysis Challenge (cMAC2) with the underlying purpose of estimating its accuracy and comparing it to the other motion estimation algorithms submitted to the challenge. The TDFFD models the velocities 
continuously in time and space as a sum of B-spline kernels. In contrast to [1], we use a similarity metric which takes into account the physics of US images. The rationale behind this new metric is that speckle remains temporally consistent for small deformations and can be used as a feature for motion tracking, while tissue and blood pool intensities are globally preserved over the cardiac cycle. Therefore, the proposed metric includes a comparison both between consecutive images (based on the correlated speckle noise as in [3]) and a comparison to the first frame (using mean square error as in [1]). We present and discuss displacement and strain quantification results on the cMAC2 dataset.

In previous work, the accuracy of the TDFFD algorithm was validated (in addition to be tested on patient data) on ground truth data with normal motion from synthetic US images [1] and tagged magnetic resonance imaging provided by the 1 st cMAC challenge [6]. Here, the data consists of synthetic images representing different configurations observable in the clinical practice, in the context of cardiac resynchronization therapy (CRT): normal heart, dyssynchronous ones with left bundle branch block (LBBB) and right bundle branch block (RBBB), and with different pacing configurations, for which dyssynchrony is expected to be reduced or corrected. The objective is to check the ability of our algorithm to estimate accurate and physiologically meaningful motion (displacement) and deformation (strain), in both healthy and pathological cases.

\section{Methods}

\section{1 cMAC2 Database}

The synthetic images combine the US imaging model described in [4] with the electro-mechanical model presented in [5]. There are in total 10 sequences simulating one normal heart as well as RBBB abd LBBB cases with and without pacing in different regions of the heart. This data includes ground truth on the motion field. For the synthetic data, volumetric meshes were provided for each frame. More details about the database and its contruction are given in the webpage of the challenge ${ }^{1}$.

In vitro images were acquired using a polyvinyl alcohol cryogel phantom and a Philips 3D X5 probe. Ground truth data was generated using microsonometry. Microsonometry measures distances and hence can be used as ground truth for radial, circumferential and longitudinal strains. Further details about the in vitro image generation can be found in the challenge webpage.

\subsection{Motion Quantification}

We use the TDFFD algorithm described in [1] to reconstruct trajectories in the cardiac cycle. The velocity field is represented as a continuous and differentiable $4 \mathrm{D}$ vector field using B-splines. The temporal smoothness of the velocity field guarantees to recover temporally smooth transformation. Another advantage is

$\overline{1}$ http://www $\cdot$ physense.org/stacom2012/ 


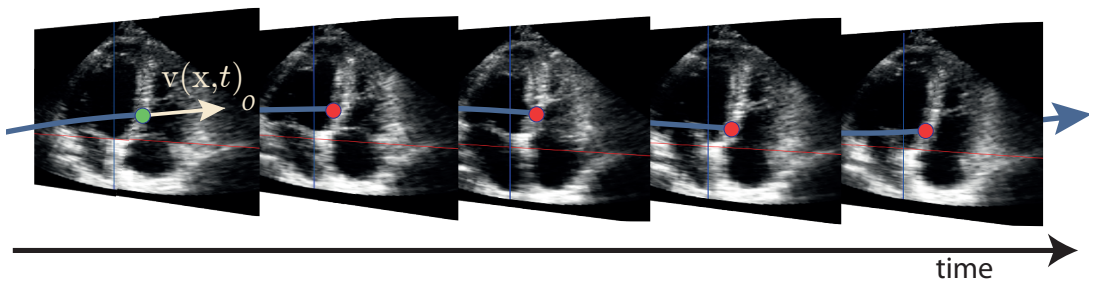

Fig. 1: The optimization of velocity at one time point depends on all previous times.

that trajectories are not restricted to the discrete set of imaging time points, thus can be evaluated for any continuous time.

The diffeomorphic mapping $\varphi: \Omega \times \mathcal{T} \rightarrow \Omega, \Omega \subset \mathbb{R}^{3}, \mathcal{T} \subset \mathbb{R}^{+}$is related to the time-varying velocity field $\mathbf{v}: \Omega \times \mathcal{T} \rightarrow \mathbb{R}^{3}$ by

$$
\boldsymbol{\varphi}_{n}^{m}(\mathbf{x} ; \mathbf{p})=\mathbf{x}+\int_{n}^{m} \mathbf{v}\left(\boldsymbol{\varphi}_{n}^{t}(\mathbf{x} ; \mathbf{p}), t ; \mathbf{p}\right) \mathrm{d} t
$$

with $\varphi_{0}^{0}=\mathbf{i d}$, where $\varphi_{m}^{n}(\mathbf{x})$ stands for the transport of a coordinate $\mathbf{x}$ at time $n$ to time $m$, and $\mathbf{p}$ is the vector of parameters (i.e., B-spline velocity coefficients assigned to all control points). At each time step, the optimization of the velocity field evaluates image intensities through all previous times, thus providing a more robust estimation. This is illustrated in Fig. 1.

The similarity metric used in [1] was the squared intensity differences between each image and the first image in the sequence. This choice was shown to avoid the accumulation of motion errors leading to drift effects. In [6], the metric was extended to include also the squared intensity differences between consecutive image pairs with the aim of improving the sensitivity to small incremental displacements. Here, we propose another approach using a combination of two similarity terms: the first one compares images over the entire cardiac cycle as in $[1,6]$, while the second one compares images at adjacent time points using an US-specific metric which considers speckle tracking information as proposed by Cohen et al. in [3]. This second metric has inherent robustness to speckle decorrelation, which makes it a suitable metric for fully-developed speckle noise.

For the metric computation, we consider two randomly drawn set of samples. The first one is $\left\{\mathbf{x}_{j} \in \Omega_{0}, j=1, \ldots, J\right\}$, where $\Omega_{0}$ is the subdomain of $\Omega$ at time $t=0$, enclosing the region of interest (e.g., the left ventricle domain). The second one is $\left\{\left(\mathbf{y}_{k}, n_{k}\right), \mathbf{y}_{k} \in \Omega_{n_{k}}, k=1, \ldots, K, n_{k} \in[1, N-1]\right\}$, where $\Omega_{n_{k}}$ is the subdomain of $\Omega$ at time $t=n_{k}$ and $N$ is the number of frames in the sequence. The proposed metric is then defined as

$M(\mathbf{p})=\sum_{j=1}^{J}\left(\Delta_{0}^{n}\left(\mathbf{x}_{j} ; \mathbf{p}\right)\right)^{2}+\lambda \sum_{k=1}^{K}\left(\ln \left(\exp 2 \Delta_{n_{k}}^{n_{k}+1}\left(\mathbf{y}_{k} ; \mathbf{p}\right)+1\right)-\Delta_{n_{k}}^{n_{k}+1}\left(\mathbf{y}_{k} ; \mathbf{p}\right)\right)$, 
where $\lambda$ is a constant factor balancing the metric terms, $\Delta_{l}^{m}(\cdot ; \mathbf{p})$ is the intensity difference between homologous points at $t=l$ and $t=m$, i.e.,

$$
\Delta_{l}^{m}(\cdot ; \mathbf{p})=I_{m}\left(\varphi_{l}^{m}(\cdot ; \mathbf{p})\right)-I_{l}(\cdot) .
$$

The second term of Eq. 2 is able to make use of speckle tracking information, whereas the first term of Eq. 2, can ensure that small errors in registration results do not add up causing significant errors over the entire cardiac cycle.

\subsection{Strain Quantification}

The strain is estimated from the spatial derivative of the reconstructed displacement field. Let $\nabla \mathbf{u}(\mathbf{x}, t)$ be the spatial gradient of displacement $\mathbf{u}(\mathbf{x}, t)$, the strain tensor can then be obtained by

$$
\boldsymbol{\epsilon}(\mathbf{x}, t)=\frac{1}{2}\left(\nabla \mathbf{u}(\mathbf{x}, t)^{T}+\nabla \mathbf{u}(\mathbf{x}, t)-\nabla \mathbf{u}(\mathbf{x}, t)^{T} \nabla \mathbf{u}(\mathbf{x}, t)\right),
$$

where superindex ${ }^{T}$ denotes transposition. The strain tensor is then projected on a set of local directions: radial, circumferential and longitudinal. The strain data is averaged over 17 regions in accordance with the standard division of the left ventricle proposed by the American Heart Association (AHA) [7]. The local directions are defined on the mesh of the first frame (corresponding to endsystole). Since strain is computed in a Lagrangian space of coordinates, local directions and AHA segments only need to be defined at the first frame.

We use centered differences on the mesh to approximate the spatial derivatives of the displacement. The radial direction is obtained from the normal to the mesh at each node of the surface. The longitudinal direction is defined as perpendicular to the radial direction while maximizing the scalar product with the apex-base vector. The circumferential direction is then obtained by the cross product of radial and longitudinal directions.

\section{Results}

Experiments were performed first on a set of synthetic 3D US sequences to evaluate the accuracy of the TDFFD algorithm with respect to known ground truth displacement. For the B-spline grid resolution, we used one control point per frame in the temporal direction and 5 control points in the short-axis and long-axis directions. The factor $\lambda$ in Eq. 2 is computed (for each sequence) as the ratio of the values of the first term of Eq. 2 to the second term, at the first iteration of the optimization process.

\subsection{Synthetic data}

For all cases, we computed the error between true and recovered displacements. The median of the peak magnitude of the difference between the ground truth 


\begin{tabular}{llc}
\hline Case & Median Peak Error (1st-3rd quartiles) \\
\hline 1 & Normal & $1.49(1.19-1.60)$ \\
8 & RBBB & $0.71(0.62-0.83)$ \\
12 & LBBB & $0.66(0.57-0.78)$ \\
20 & LBBB+Pacing 5 & $0.76(0.65-0.85)$ \\
22 & LBBB+Pacing 6 & $1.38(1.12-1.48)$ \\
28 & LBBB+Pacing 7 & $0.80(0.66-0.87)$ \\
36 & LBBB+Pacing 12 & $0.78(0.60-0.92)$ \\
44 & RBBB+Pacing 9 & $0.74(0.67-0.78)$ \\
60 & LBBB+Pacing 3+6 & $0.71(0.63-0.82)$ \\
88 & LBBB+Pacing $7+14$ & $0.71(0.66-0.79)$ \\
\hline
\end{tabular}

Table 1: Median (with first and third quartiles) of the peak errors (in mm) of the proposed image registration for each case in the dataset.

displacement field and the one obtained by our algorithm are summarized in Table 1 for each case. The median peak error was obtained by computing the median of the maximum error at each AHA segment.

Fig. 2 plots the mean registration error (over all cases) for each segment and direction. Fig. 3 shows displacement magnitude and circumferential strain for cases 1 (normal), 12 (LBBB) and 22 (LBBB with pacing at AHA segment 6) for the AHA segments at the septum and lateral wall. Displacement magnitudes for case 12 are reduced with respect to the other cases. Moreover, dyssynchrony can be observed for this case between septal segments (2, 3, 8 and 9) and lateral segments $(5,6,11$ and 12$)$, which contract about $20 \%$ of the cycle later. When looking at displacements for case 22 , this temporal difference in reduced for almost $10 \%$ of the cycle and displacement results are similar to the ones obtained for case 1 . As for circumferential strain, while rather uniform strain patterns are observed for the normal case, an overall reduction of its magnitude is found for case 12 , and its value is almost zero for septal segments during the whole cycle (probably due to the LBBB). In case 22, circumferential strain magnitude is increased with respect to case 12 and more similar to case 1, presenting contraction at septal segments.

\subsection{Phantom data}

On phantom data, ground truth was provided as distance between pairs of sonomicrometry crystals. These distances give an approximation of radial, circumferential and longitudinal strains. Crystals positions were provided in the image space of coordinates. We computed the trajectories and the pairwise distances corresponding to those given by the ground truth.

Fig. 4 plots on the top row the three strains as provided by sonomicrometry for each acquisition, and on the bottom row the ones obtained by our TDFFD algorithm. As expected, the magnitude of global deformation is reduced with compression. Radial strain is almost zero and, unlike in myocardial tissue, the longitudinal strain takes positive values. One can see that circumferential and 

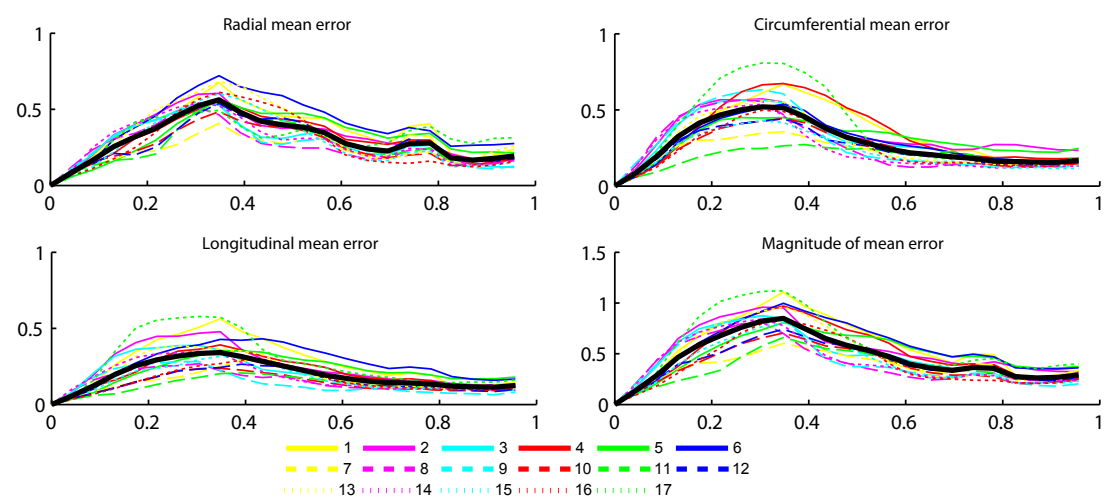

Fig. 2: Segmental displacement error (in millimiters), average over all cases. From top to bottom and from left to right: radial, circumferential, longitudinal and overall. Thick black curve corresponds to the median error for all segments. The horizontal axis is the normalized cardiac time (from 0 to 1 ).
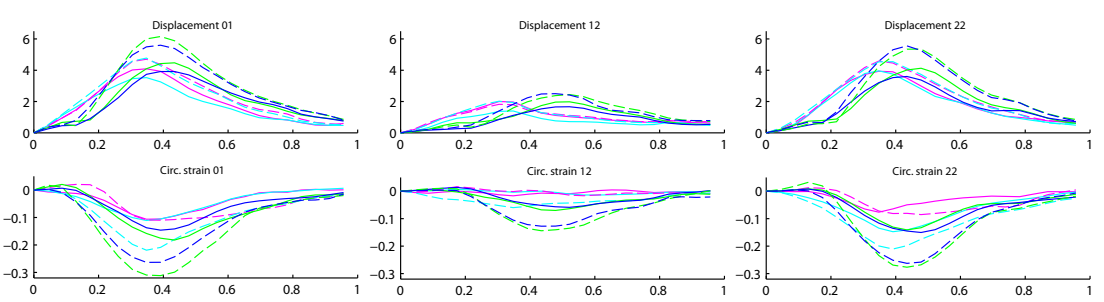

Fig. 3: Displacement (top) and circumferential strain (bottom) for case 1 (normal), 12 (LBBB) and 22 (LBBB with pacing at AHA segment 6) for septum and lateral wall segments. Thick black curve corresponds to the median strain for all segments. The horizontal axis is the normalized cardiac time (from 0 to 1 ).

longitudinal strain curves from ground truth and from our algorithn are similar in amplitude and shape.

\section{Conclusions}

In this paper, the TDFFD algorithm was extended to include an US-specific metric that considers the correlated speckle noise between consecutive images and has inherent robustness to speckle decorrelation. The use of both this sequential metric and the non-sequential one results in a good compromise between low error values over the contraction period and low temporal drifts in the last phases. Preliminar motion and deformation results were reported for the phantom data provided by the cMAC2. Overall, mean displacement error was below $1 \mathrm{~mm}$. Uniform strain patterns were observed over all myocardial segments for the normal case, as physiologically expected. Comparing the normal with the 


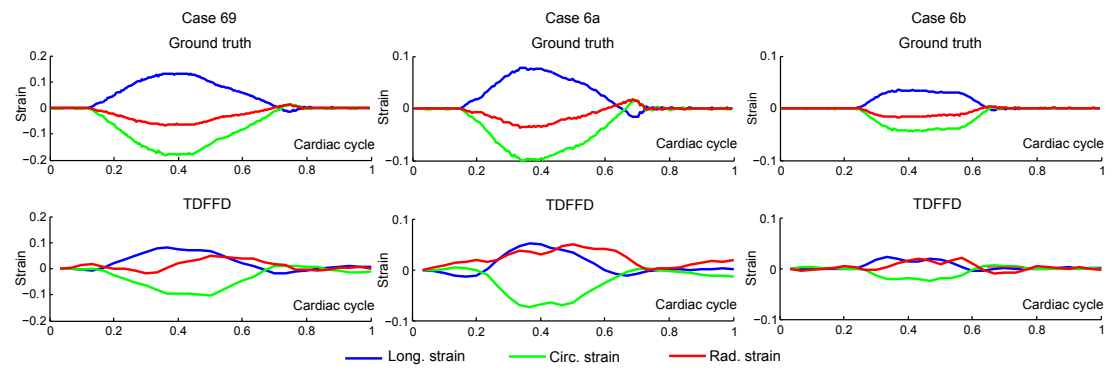

Fig. 4: Strain in radial, circumferential and longitudinal directions from sonomicrometry crystals (top) and from tracking on the 3D US sequences (bottom). The horizontal axis is the normalized cardiac time (from 0 to 1 ).

LBBB case (with and without pacing), the capability to recover dyssynchrony as assessed by the ground truth it is shown.

\section{Acknowledgments}

This research has been partially funded by the Spanish Industrial and Technological Development Center (CDTI) under the CENIT Programme (cvREMOD Project), the Spanish Ministry of Science and Innovation (Ref. TIN2009-14536-C02-01), Plan E and FEDER, and the European Community's Seventh Framework Program (FP7/20072013) under the euHeart project (FP7-ICT-2007-2-224495). ARP is funded by the Spanish Government with a FPU grant.

\section{References}

1. De Craene, M., Piella, G., Camara, O., Duchateau, N., Silva, E., Doltra, A., D'hooge, J., Brugada, J., Sitges, M., Frangi, A.F.: Temporal diffeomorphic free-form deformation: application to motion and strain estimation from 3D echocardiography. Med Image Anal 16(2) (2012) 427 - 450

2. De Craene, Piella, G.: An implementation of the TDFFD and LDFFD algorithms. Insight Journal 2012. http://hdl.handle.net/10380/3345

3. Cohen, B., Dinstein, I.: New maximum likelihood motion estimation schemes for noisy ultrasound images. Pattern Recogn 35(2) (2002) 455-463

4. Gao, H., Choi, H.F., Claus, P., Boonen, S., Jaecques, S., van Lenthe, G., Van Der Perre, G., Lauriks, W., D'hooge, J.: A fast convolution-based methodology to simulate 2-D/3-D cardiac ultrasound images. IEEE Trans Ultrason, Ferroelect, Freq Contr 56(2) (2009) $404-409$

5. Sermesant, M., Chabiniok, R., Chinchapatnam, P., Mansi, T., Billet, F., Moireau, P., Peyrat, J., Wong, K., Relan, J., Rhode, K., Ginks, M., Lambiase, P., Delingette, H., Sorine, M., Rinaldi, C., Chapelle, D., Razavi, R., Ayache, N.: Patient-specific electromechanical models of the heart for the prediction of pacing acute effects in crt: A preliminary clinical validation. Med Image Anal 16(1) (2012) $201-215$ 
This a pre-print version.

The final document is available at http://www.springerlink.com

6. De Craene, M., Tobon-Gomez, C., Butakoff, C., Duchateau, N., Piella, G., Rhode, K.S., Frangi, A.F.: Temporal diffeomorphic free form deformation (TDFFD) applied to motion and deformation quantification of tagged MRI sequences. In Camara, O., Konukoglu, E., Pop, M., Rhode, K.S., Sermesant, M., Young, A.A., eds.: Statistical Atlases and Computational Models of the Heart. Imaging and Modelling Challenges, at International Conference on Medical Image Computing and Computer-Assisted Intervention (MICCAI). Volume 7085 of LNCS., Springer (2011) $68-77$

7. Cerqueira, M.D., Weissman, N.J., Dilsizian, V., Jacobs, A.K., Kaul, S., Laskey, W.K., Pennell, D.J., Rumberger, J.A., Ryan, T., Verani, M.S.: Standardized myocardial segmentation and nomenclature for tomographic imaging of the heart: a statement for healthcare professionals from the cardiac imaging committee of the council on clinical cardiology of the American Heart Association. Circulation 105(4) (2002) 539-542 